\title{
Scalable High-Gaussicity Split-Block Diagonal Horn Antenna for Integration with Sub-THz Devices
}

\author{
H. M. Santos ${ }^{1,2}$, E. D. Lima ${ }^{1}$, Pedro Pinho ${ }^{3,4}$, L. M. Pessoa ${ }^{1}$, D. Moro-Melgar ${ }^{5}$, H. M. Salgado ${ }^{1,2}$ \\ ${ }^{1}$ INESC TEC, Porto, Portugal \\ ${ }^{2}$ Faculdade de Engenharia da Universidade do Porto, Portugal \\ ${ }^{3}$ Instituto de Telecomunicações \\ ${ }^{4}$ ADEETC, Instituto Superior de Engenharia de Lisboa \\ ${ }^{5}$ ACST GmbH, Hanau, Germany \\ ${ }^{1}$ \{hugo.m.santos, erick.d.lima, luis.m.pessoa, henrique.salgado\}@ @inesctec.pt, ${ }^{4}$ ppinho@ deetc.isel.pt, ${ }^{5}$ diego.moro-melgar@acst.de
}

\begin{abstract}
In this paper we propose a high-gaussicity spline-profiled horn antenna, which is scalable in length and aperture to achieve higher gains whilst retaining a high Gaussian efficiency. A novel approach is used where a PSO is used for optimizing the spline, using the gaussicities at the operating frequencies as the objective function, which further improves side-lobe level and cross-polarization when compared to the state-of-the-art. With the proposed method, which was validated through FEM simulations in HFSS, reflection coefficients below $-15 \mathrm{~dB}$, gains greater than $25 \mathrm{dBi}$ and gaussicities above $91 \%$ were obtained in the entire WR-3 band.
\end{abstract}

Keywords - Horn, Gaussicity, Split-Block, Terahertz

\section{INTRODUCTION}

The gaussicity of an antenna is an important parameter, when their radiated beam is meant to go through quasi-optical processing, i.e. when the beam is meant to pass through multiple reflectors, lenses, polarizing grids, among others [1].

Typically, the corrugated horn is the choice of election to achieve a high Gaussian beam coupling efficiency. [2], [3]. Despite the fact that this type of horn antenna has been extensively studied as an efficient Gaussian beam launcher, when frequency increases this approach becomes intricate due to the available fabrication processes.

The split-block technique appeared as an alternative to the conventional fabrication methods. The diagonal horn is a case in which the fabrication technique fits this category. It has been theoretically studied by the decomposition in Gaussian-Hermite modes by Johansson and Whyborn in 1992 [4]. However, the gaussicity achieved by the diagonal horn antenna is approximately $84 \%$ which is less than the typical $98 \%$ of corrugated horns or $96.3 \%$ of potter horns [1].

The smoothed-walled variable profile horn appeared as a viable alternative to the diagonal horn, in which the cutting tool does not follow a continuous profile when passing through the split-block. Instead, cutting depth variations are present which were proven to achieve high gaussicities [5], [6]. In [6] a spline-profiled diagonal horn was proposed and gaussicities of approximately $97 \%$ were obtained at the centre frequency, through an optimization process. However, horn scalability analysis for achieving higher gains was not accomplished.
Additionally, due to the fact that gaussicity was not part of the optimization process, the cross-polarization, side-lobe level and gaussicities might be further improved.

In this paper a Particle Swarm Optimizer (PSO) was employed to determine the optimal spline dimensions relative to the diagonal horn aperture. This allows a scalable empirical model to be built, similar to the one verified in [7] for conical horns. The model which is scaled in terms of horn aperture and length, guides the designer on how to control the gain and still retain the high gaussicity of the split-block spline-profiled diagonal horn antenna. A comparison can be seen in Table 1, in which it can be seen that using the gaussicity at key points in the WR-3 band results in improved performance. Additionally this performance is attained in the whole WR- 3 band rather than only in portions of the operating bandwidths as it is the case in other works.

Table 1. Comparison table with worst sidelobe level and cross polarization discrimination (XPD).

\begin{tabular}{ccc}
\hline Reference & Worst Sidelobe Level $(\mathrm{dB})$ & Worst XPD $(\mathrm{dB})$ \\
\hline$[5]$ & -40 & 36.86 \\
{$[6]$} & -30 & 24 \\
This Work & -36 & 40
\end{tabular}

The paper is organized as follows: the next section addresses the preliminary antenna design procedure, section III presents the scalability formulation and analysis of the preliminary design and section IV demonstrates the final design. Section V provides the conclusions and guidelines for future work.

\section{Preliminary Antenna Design}

The design of the split-block diagonal horn antenna was made taking into consideration the model of Fig. 1, in which a top view of the split-block is shown. The spline depths $x_{i}$, where $i$ is the index of the point of the spline are given by $x_{i}=k_{i} \cdot d$. The dimension $d$ corresponds to the diagonal horn aperture and $l$ to the horn length. The $\mathrm{H}$-wall of the waveguide is given by $b=0.4318 \mathrm{~mm}$ for the case of the WR-3 interface considered in this work. 


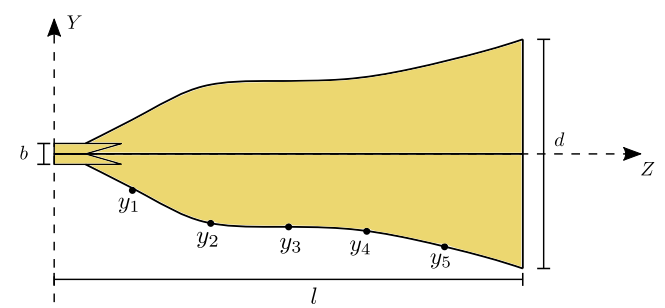

Fig. 1. Preliminary antenna model (top view of one of the two split-blocks).

Since the waveguide interface has fixed dimensions, the PSO algorithm can act in variables $d, l$ and $k_{i}$, which in turn will define variables $x_{i}$. For simulation time reduction, a fixed horn length of $l=10 \mathrm{~mm}$ was considered. The remaining variables $k_{i}$ and $d$ were used as the search space of the algorithm. Due to fabrication limitations, for spline depths that were not continuously decreasing, the solutions were discarded by the PSO. The boundaries of the search space are described in Table 2.

Table 2. Solution space boundaries considered for the PSO algorithm.

\begin{tabular}{ccc}
\hline Variable & Lower Bound & Upper Bound \\
\hline$k_{1}$ & 0.03 & 0.2 \\
$k_{2}$ & 0.08 & 0.25 \\
$k_{3}$ & 0.18 & 0.3 \\
$k_{4}$ & 0.2 & 0.4 \\
$k_{5}$ & 0.3 & 0.5 \\
$d$ & $5 \mathrm{~mm}$ & $8 \mathrm{~mm}$
\end{tabular}

The remaining PSO parameters, namely the cognitive coefficient $c 1=1.6$, the social coefficient $c 2=1.6$ and the inertia coefficient $\omega=0.3$, were defined considering the recommendations of [8]. The number of particles (candidate solutions) was defined as $N=100$, since according to [8] this will lead to a reduced number of iterations and potentiate a better search of the solution space. The maximum number of iterations was set to 100 , but a stopping condition was defined if the best objective function $O F$ value suffered no change in 5 consecutive iterations.

The $O F$ value was calculated by considering the geometric mean of the gaussicities $\eta_{g 1}, \eta_{g 2}$ and $\eta_{g 3}$ at $220 \mathrm{GHz}, 275 \mathrm{GHz}$ and $330 \mathrm{GHz}$, respectively, written as

$$
O F=\sqrt[3]{\eta_{g 1} \cdot \eta_{g 2} \cdot \eta_{g 3}}
$$

where the three gaussicities are used so that acceptable Gaussian beam launching capabilities are obtained in the full WR-3 band. The PSO algorithm was modified so that it would maximize the value of the objective function, instead of minimizing it.

Each gaussicity was calculated by the formula [1]

$$
\eta_{g}(\%)=100 \times \frac{\left|\iint_{A} \vec{E} \cdot \overrightarrow{g^{*}} d A\right|^{2}}{\iint_{A} \vec{E} \cdot \overrightarrow{E^{*}} d A \cdot \iint_{A} \vec{g} \cdot \overrightarrow{g^{*}} d A}
$$

in which $A$ represents the area over the horn aperture and $\vec{E}$ the electric field at the aperture. The fitting Gaussian curve, $\vec{g}$, is given by

$$
\vec{g}(x, y)=e^{-\left(\frac{\sqrt{x^{2}+y^{2}}}{w}\right)^{2}} \hat{u}
$$

where $x$ and $y$ correspond to the coordinates of the horn aperture. The co-polarization unit vector is denoted as $\hat{u}$ and $w$ represents the Gaussian beam radius, which equivalently corresponds to the standard deviation and is the parameter enabling the fitting of the Gaussian curve to the calculated E-field. The standard deviation was calculated by taking into account the full width at half-maximum, $F W H M$, of the co-polarized E-field along the $X$ and $Y$ axes at the horn aperture. The radius $w \mathrm{w}$ is calculated as

$$
w=\sqrt{\sigma_{X} \cdot \sigma_{Y}}
$$

and relating the standard deviation in terms of the $F W H M$, yields

$$
\sigma_{X, Y}=\frac{F W H M_{X, Y}^{2}}{4 \ln (2)}
$$

The method for calculating the gaussicity was checked by simulating the diagonal horn reported in [4] and comparing the calculated gaussicity of $83.7 \%$ with the reported theoretical of $84.3 \%$. Since the values were in close agreement, the method was validated.

After running the PSO algorithm, we obtained the convergence plot of Fig. 2, in which we can see that after 22 iterations, the objective function value settled around $O F \approx$ 93. The variables which correspond to the optimal solution are $k_{1}=0.140, k_{2}=0.186, k_{3}=0.192, k_{4}=0.240$, $k_{5}=0.367$ and $d=7.410 \mathrm{~mm}$. The calculated gaussicities of this optimized horn antenna were $\eta_{g 1}=91.13 \%$ at $220 \mathrm{GHz}$, $\eta_{g 2}=93.12 \%$ at $270 \mathrm{GHz}$ and $\eta_{g 3}=93.84 \%$ at $330 \mathrm{GHz}$. A peak gain of $20 \mathrm{dBi}$ was obtained at $275 \mathrm{GHz}$. However, if more or less gain is required, the scaling of the proposed antenna is necessary. Such analysis is presented in the next section.

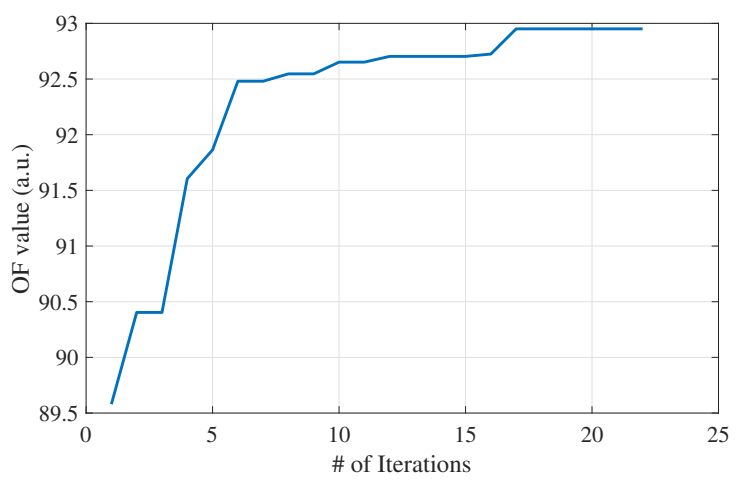

Fig. 2. PSO convergence evolution with number of iterations. 


\section{SCALABILITY ANALYSIS}

The scaling of the proposed horn antenna allows for a more flexible design depending on the needs in terms of gaussicity and gain. This analysis was done by sweeping the horn length, $l$, and its aperture, $d$, as given in the previous section. The horn length, $l$ was swept from $3 \lambda$ to $40 \lambda$, so that the analysis is valid when lower or higher gains are needed, respectively. Due to the fact that the field in the horn aperture is similar to the field of a conical horn $\left(\mathrm{TE}_{11}\right)$, the fitting functions used were analogous to the ones used in the analysis of conical horns made in [7].

The first step of the scalability analysis was to determine the horn aperture that corresponds to the optimal gaussicity as a function of the horn length and wavelength considered for the gaussicity calculation. A square root function was used for curve fitting, which resulted in an optimal horn aperture given by the fitting function

$$
d=\sqrt{5.15 \cdot l \cdot \lambda}
$$

which is plotted in Fig. 3, alongside with the simulation data.

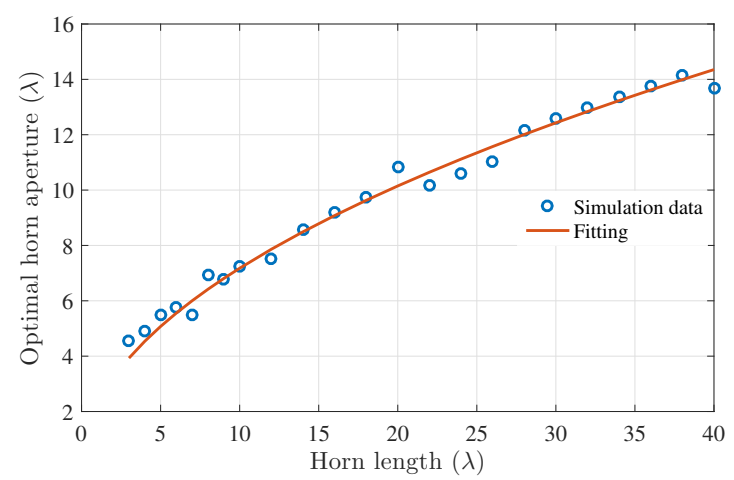

Fig. 3. Optimal horn aperture as a function of horn length.

The scaling process under analysis is meant to allow the control of the gain of the antenna. Therefore, we analysed the gain of the proposed horn as a function of its length. Despite the fact that in [7] the analysis for conical horns is made in terms of the aperture, we used the length for our analysis because the optimal aperture is already being considered and its relation with the horn length is known. The gain in $\mathrm{dBi}$ of the horn as a function of its length was fitted to simulated data using

$$
G(\mathrm{dBi})=10 \log _{10}\left(9.486 \cdot \frac{l}{\lambda}\right)
$$

The fitting function and the simulated data are plotted in Fig. 4.

Due to the fact that the horn aperture is optimal for a specific wavelength and horn length, the gaussicity and gain will vary with frequency. Since the optimal aperture was considered to obtain peak gaussicity at a specific frequency, it is expected that this parameter will decrease for higher and lower frequencies. The scaling process that was derived is

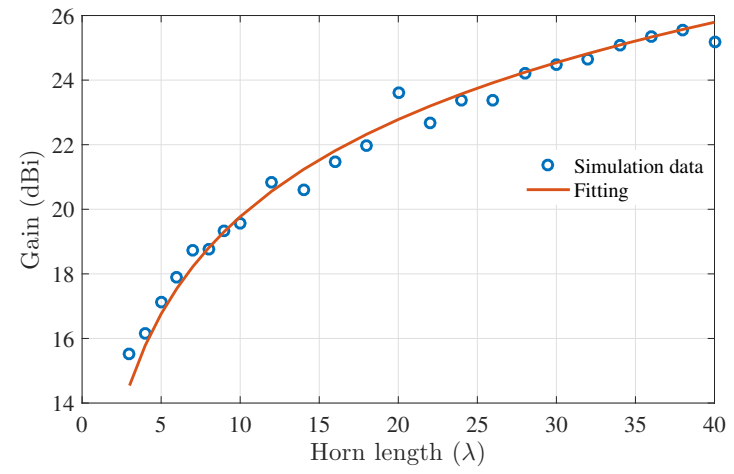

Fig. 4. Horn gain as a function of horn length, considering optimal horn aperture.

employed for the design of a spline-profiled horn, with optimal gaussicity at $275 \mathrm{GHz}$, in the next section.

\section{FINAL DESIGN}

The requirements of the horn were set having in mind a final application where the antenna is to be integrated with Schottky barrier diodes and are: a gain of at least $25 \mathrm{dBi}$ and a gaussicity as high as possible in the entire WR-3 band.

Considering the scalable model derived in section III, it is possible to infer that the gain increases with increasing frequency. Therefore the $25 \mathrm{dBi}$ gain restriction must be fulfilled at $220 \mathrm{GHz}$, the lowest frequency of the WR-3 band. Plugging these values into equation (7), a horn length of $l \approx 45 \mathrm{~mm}$ was obtained. Given the horn length, we can calculate the aperture that results in maximum gaussicity at the centre frequency of $275 \mathrm{GHz}$.Additionally, the use of equation (6) gives an horn aperture of $d \approx 15.9 \mathrm{~mm}$.

For computing cross-polarization patterns and better approximate the real behaviour of the physical antenna to be fabricated, a simulation of the full antenna model had to be performed. A finite conductivity of $\sigma=2 \times 10^{7} \mathrm{~S} / \mathrm{m}$ was considered for the waveguide and horn walls which corresponds to the gold alloy that will be used in the manufacturing process.

After simulation of the horn antenna, the input reflection coefficient was obtained as shown in Fig. 6. In the WR-3 band the $S_{11}$ magnitude stays below $-15 \mathrm{~dB}$ which allows us to state that the antenna is suitable for operation.

The co- and cross-polarization radiation patterns are shown in Fig. 5. The XPD can be seen to be greater than $40 \mathrm{~dB}$ at all frequencies in the theta scan range where most of the radiation takes place. Nonetheless, it degrades for angles farther away from the desired radiation direction. These results show the advantage of using the gaussicity of the horn as the objective function to adjust its spline profile compared to when multiple optimization goals such as low side-lobes and reduced cross-polarization are used [6]. In Table 3 the gains, gaussicities and phase centre offset from the horn aperture to the inside of the horn are shown. The gains are not only in compliance with the design requirements but also agree 


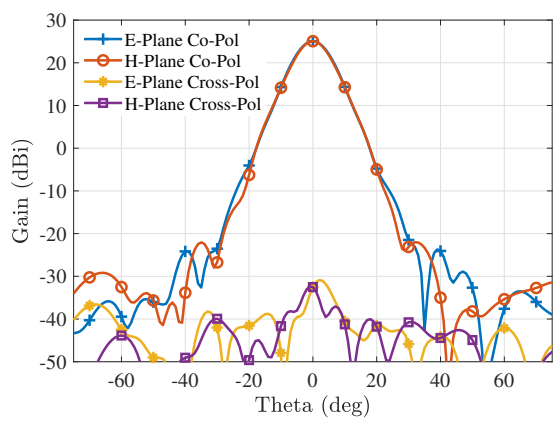

(a)

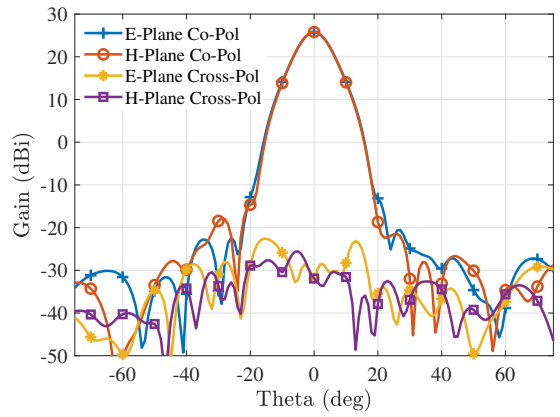

(b)

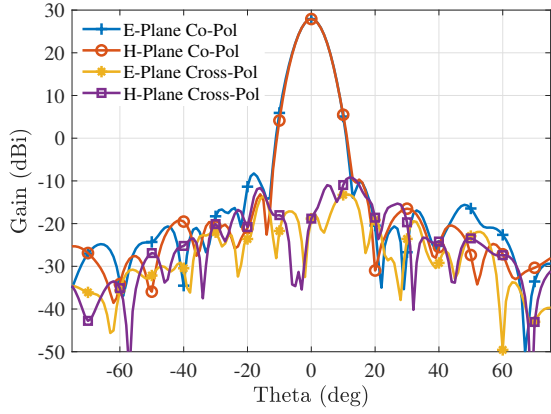

(c)

Fig. 5. Simulated E-plane and H-plane co- and cross-polarization radiation patterns at (a) $220 \mathrm{GHz}$, (b) $275 \mathrm{GHz}$ and (c) $330 \mathrm{GHz}$.

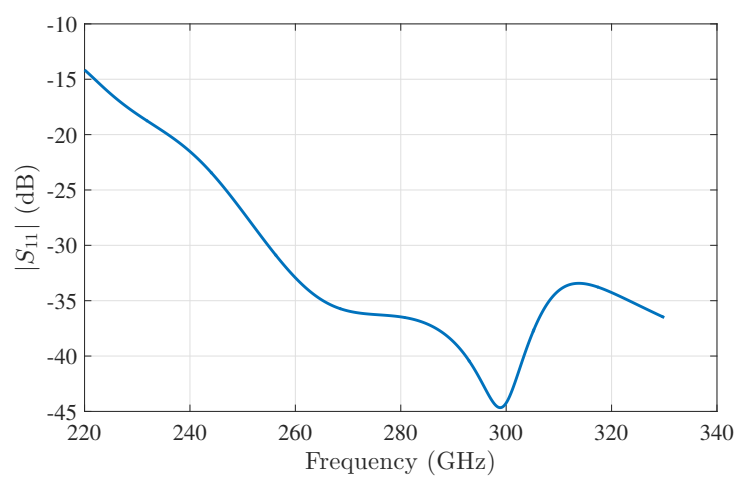

Fig. 6. $\left|S_{11}\right|$ as a function of frequency for the full model simulation.

with the ones that can be obtained from the model derived in section III. The gaussicity peaks at the centre frequency of $275 \mathrm{GHz}$ and retains a high value in the WR-3 band. A full design of one of the split-blocks is shown in Fig. 7 where the mounting flange and screws are also visible.

Table 3. Field parameters for the start, centre and end frequencies of WR-3 band.

\begin{tabular}{cccc}
\hline Parameter & $220 \mathrm{GHz}$ & $275 \mathrm{GHz}$ & $330 \mathrm{GHz}$ \\
\hline$\eta_{g}$ & $91.4 \%$ & $95.9 \%$ & $91.1 \%$ \\
Gain & $25.1 \mathrm{dBi}$ & $25.8 \mathrm{dBi}$ & $27.9 \mathrm{dBi}$ \\
PC $_{\text {offet }}$ & $20.9 \mathrm{~mm}$ & $19.4 \mathrm{~mm}$ & $15.8 \mathrm{~mm}$
\end{tabular}

\section{CONCLUSiOn}

In this paper, a spline-profiled horn was presented, for which a full scalability analysis was performed and validated through FEM simulations in HFSS. It was proven that by using the gaussicities in the operating frequencies as the objective function for optimizing the horn geometry, better results can be obtained, compared to when multiple optimization goals are used such as low side-lobes and reduced cross-polarization.

As future work the we will validate the results obtained here through measurements of the fabricated antenna.

\section{ACKNOWLEDGEMENT}

The research leading to these results has received funding from the European Union's Horizon 2020 - The EU Framework

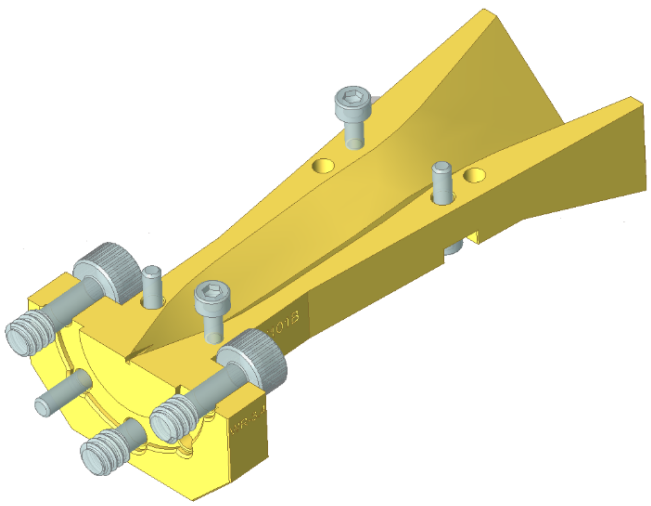

Fig. 7. 3D model ready for fabrication of the proposed horn.

Programme for Research and Innovation 2014-2020, under grant agreement No 761579, and from FCT (Fundação para a Ciência e a Tecnologia, Portugal), through the Ph.D. grant PD/BD/128197/2016.

\section{REFERENCES}

[1] J. F. Johansson, "A Comparison of Some Feed Types," in Astronomical Society of the Pacific Conference Series, vol. 75, May 1994, pp. 82-89.

[2] R. J. Wylde, "Millimetre-wave Gaussian beam-mode optics and corrugated feed horns," IEE Proceedings H - Microwaves, Optics and Antennas, vol. 131, no. 4, pp. 258-262, August 1984.

[3] J. E. McKay, D. A. Robertson, P. J. Speirs, R. I. Hunter, R. J. Wylde, and G. M. Smith, "Compact Corrugated Feedhorns With High Gaussian Coupling Efficiency and-60 dB Sidelobes," IEEE Transactions on Antennas and Propagation, vol. 64, no. 6, pp. 2518-2522, June 2016.

[4] J. F. Johansson and N. D. Whyborn, "The diagonal horn as a sub-millimeter wave antenna," IEEE Transactions on Microwave Theory and Techniques, vol. 40, no. 5, pp. 795-800, May 1992.

[5] D. McCarthy, J. A. M. Neil Trappe, C. O'Sullivan, M. Gradziel, S. Doherty, C. Bracken, N. Tynan, A. Polegre, and P. Huggard, "Efficient algorithms for optimising the optical performance of profiled smooth walled horns for future CMB and Far-IR missions," vol. 9153, 2014, pp. $9153-9153-8$.

[6] H. J. Gibson, B. Thomas, L. Rolo, M. C. Wiedner, A. E. Maestrini, and P. de Maagt, "A novel spline-profile diagonal horn suitable for integration into thz split-block components," IEEE Transactions on Terahertz Science and Technology, vol. 7, no. 6, pp. 657-663, Nov 2017.

[7] C. Balanis, Antenna Theory: Analysis and Design. Wiley, 2016.

[8] F. Marini and B. Walczak, "Particle swarm optimization (PSO). A tutorial," Chemometrics and Intelligent Laboratory Systems, vol. 149, pp. $153-165,2015$. 